\title{
Application of Multi-Contour Cutting in Algorithms for Solving the Cutting Path Problem
}

\author{
Anastasiia Tavaeva ${ }^{2,1,}$, Aleksandr Petunin ${ }^{1}$, and Efim Polishchuk ${ }^{1}$ \\ ${ }^{1}$ Ural Federal University, 620002 Ekaterinburg, 19 Mira st., Russia \\ ${ }^{2}$ Joint-Stock Company " Production Association "Ural Optical and Mechanical Plant named after Mr. \\ E.S. Yalamov”, 620100 Ekaterinburg, 33B Vostochnaya St., Russia
}

\begin{abstract}
The cutting path problem for $\mathrm{CNC}$ sheet metal cutting equipment is considered in this article. The method of multi-contour cutting application is described for solving Cutting Path Problem (CPP). The method is to be combine contours in «blocks» (groups) and cutting of several contours is performed by using special cutting techniques (common cut, «chained» cutting) in order to reduce values of cutting tool length and number of piercings compared with standard cutting technique (where only idle path is minimized) The article presents results of solving cutting path problem computing experiments using proposed method (multi-contour cutting with common cut) and algorithm CCP-Relax (in which the standard cutting technique is used). The examples of testing are performed by using several instances from Library CPPLib.
\end{abstract}

\section{Introduction}

The Cutting Path Problem (CPP) is a complex optimization problem that occurs in developing of numerical control (NC) programs for CNC technologies equipment. The NC programs contain information about tool path of $\mathrm{CNC}$ equipment and some technological commands. Optimization of tool path minimises cutting time and cost. The goal of the CPP is to minimize cost and time of cutting process associated with cutting of required parts contours from sheet metal.

As optimization criteria (objective functions) in CPP the cutting cost $F_{\cos t}$ and time $T_{\text {cut }}$ are considered $[1,2]$ :

$$
\begin{aligned}
& F_{\mathrm{cos} t}=L_{o n} \cdot C_{o n}+L_{o f f} \cdot C_{o f f}+N_{p t} \cdot C_{p t}, \\
& T_{\mathrm{c} u t}=\frac{L_{o n}}{V_{o n}}+\frac{L_{o f f}}{V_{o f f}}+N_{p t} \cdot t_{p t} .
\end{aligned}
$$

\footnotetext{
*Corresponding author: tavaeva_a_f@bk.ru, a.f.tavaeva@urfu.ru
} 
Here $L_{o n}$ is length of cutting tool path; $L_{\text {off }}$ is length of idle tool path; $C_{o n}$ is unit cost of cutting tool path; $C_{\text {off }}$ is unit cost of idle tool path; $N_{p t}$ is numbers of piercings; $V_{o n}$ is speed of cutting tool path; $V_{\text {off }}$ is speed of idle tool path; $t_{p t}$ is time of one piercing; $C_{p t}$ is cost of one piercing.

At present the following classes of Cutting Path Problem is considered depending on using cutting technique and its parameters [3-5]:

- Traveling Salesman Problem (TSP). The tool can enter and exit contours at one point and the contour must be cut completely before tool moves to the next contour;

- Generalized Traveling Salesman Problem (GTSP).The parts are cut out sequentially and tool visits each contour to be cut once. The tool can enter and exit contours at some predefined points on the boundary;

- Continuous Cutting Problem (CCP). The parts are cut out sequentially; the standard cutting technique is used (when numbers of contours equal numbers of piercing). The cutting tool visits each contour to be cut once. The tool can enter the contour at any point on its perimeter, but must cut the entire contour before one travels to the next contour;

- Endpoint Cutting Problem (ECP). The tool can enter and exit contours at some predefined points on the boundary. In this case the contours may be cut in sections using special cutting techniques;

- Segment Continuous Cutting Problem (SCCP). In this case the notion of segment is introduced. The segment may be section of the contour or combining several contours and/or their sections. Each segment is cut completely, consequently, $C C P \subset S C C P$;

- Generalized Segment Continuous Cutting Problem (GSCCP). This problem similar to the SCCP, but segmentation is not set in advance and it is subject to optimization itself;

- Intermittent Cutting Problem (ICP). This is the most general version of the problem in which the contours can be cut by sections and tool can enter the contour at any point on its perimeter.

The difference between the problem classes under consideration is due to different rules for choosing possible piercings and cutting methods. In the most of articles dedicated designing of cutting path for sheet metal $\mathrm{CNC}$ technologies equipment, the methods and corresponding them algorithms. This algorithms usually based on application of standard cutting technique. Thus the cutting path problem is reduced to problem only of idle tool motion optimization. In overwhelming part of articles on the research issue the discretization of the boundary contours is used [6-13], consequently the various mathematical models of discreet optimization may be used. We can only mention some articles where optimization algorithms are focused on finding solutions among continuum sets [14-17]. Generally it should be noted disadvantages of research about problems of special cutting techniques application and methods of their use in design of cutting path in CAM. There are special cutting techniques - common cut, «chained» cutting, «snake» cutting, et al [5]. The use of common cut is useful during cutting of high-cost sheet material on CNC equipment because a reusable scrap is generated.

In this article the problem of multi-contour cutting application with common cut is discussed, which is weakly covered in open sources. 


\section{A new method of multi-contour cutting application for solving the Cutting Path Problem}

As mentioned before the standard cutting technique is often used during design of NC programs for $\mathrm{CNC}$ sheet cutting equipment. In the case of standard cutting the contour is cut by one piercing and tool moving from one contour to another is possible after the final cutting of previous one (fig.1a). Thus in this problem definition the cutting cost or time minimization is possible only from the point of idle tool movement minimization. As is well known [2], the piercing cost and unit cost of cutting tool path can tens of times higher than unit cost of idle tool path. Consequently, application of special cutting techniques for cutting path generation is actual issue at present. Currently special cutting techniques include multi-contour and multi-segment cutting. In the case of multi-segment cutting only part of the contour is contained, in the case of multi-contour cutting - several contours (or their parts).

Definition 1. Cutting segment $S$ - is a tool trajectory from piercing M to appropriate point of switching off tool $\mathrm{M}^{*}$.

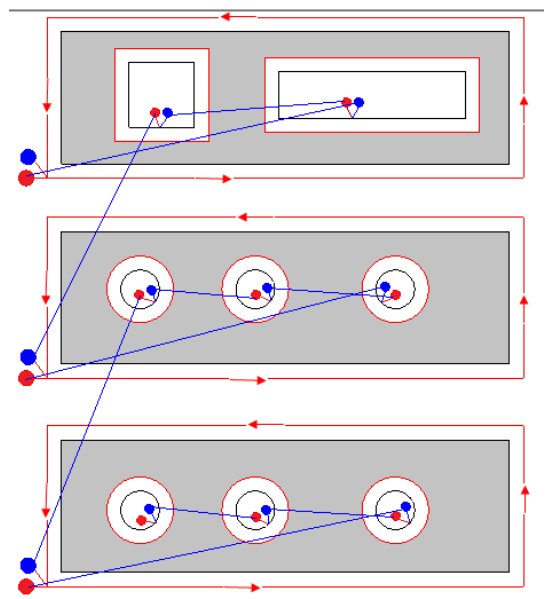

a)

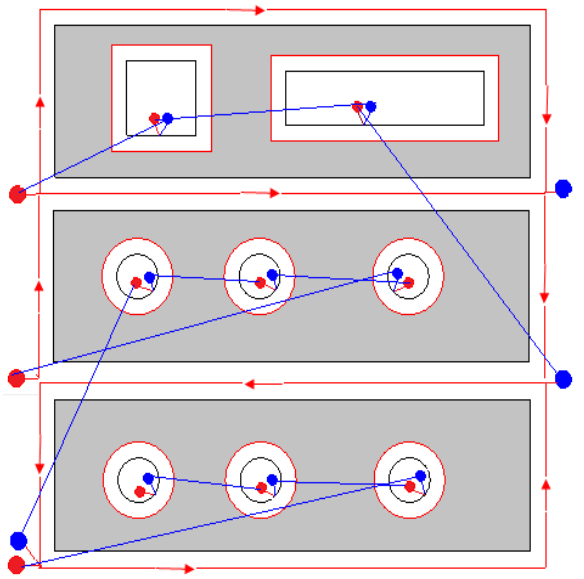

b)

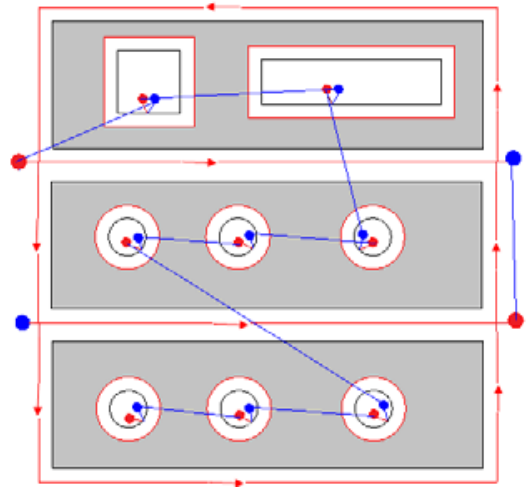

c)

Fig. 1. The example of cutting techniques application for rectangular parts. 
The method of cutting segments generation for rectangular parts is proposed in this article, i.e. the outer contour is rectangular, at the same time the inner contours are allowed. Key part of the proposed method is to combine parts into groups or «blocks» and application of common cut technique for generation of cutting tool path in each group in order to reduce values of basic cutting parameters - numbers of piercings and length of cutting tool path that finally results in reducing of objective function value. Fig. 1a presents example of cutting path generation for rectangle parts using standard cutting technique, fig. $1 \mathrm{~b}$ presents multi-segment cutting, fig.1c presents multi-contour cutting of parts using proposed in this article method (namely parts are combined in groups and inside the group the cutting of 3 contours takes place with cutting pass using two piercing). Thus in the case of fig. $1 \mathrm{a}$ the outrer contours is cut by using 3 segments, in the case of fig. $1 \mathrm{~b}$ - for three segments, number of piercings equels number of piercing during standard cutting, but value of $L_{o n}$ is reduced by common cuts, in the case of fig.1c - for two segments, numbers of piercing and value of $L_{\text {on }}$ are less than in case of fig.1a,b due to common cuts.

The rectangular parts can be nested in several rows by method proposed in fig.1c,2 and using this method the value of objective function may be significantly reduced. As a result of parts combine and the subsequent cutting contours by proposed method (fig.1c, 2), the number of piercings compared to standard cutting will be reduced to:

$$
N_{p t}=p+(l-2),
$$

At the same time value of cutting path length $L_{\text {on }}^{\text {common }}$ by placing parts with method presented in fig.1c, 2, compared with using of standard cutting technique will be reduced to:

$$
L_{\text {on }}^{\text {common }}=L_{\text {on }}^{\text {st }}-a \cdot p \cdot(l-1)-b \cdot l \cdot(p-1),
$$

where $p$-number of rectangle parts in «block» at a horizontal row (fig.2), $l$-number of rectangle parts in «block» at a vertical row (fig.2), $L_{o n}^{s t}$ - length of cutting tool path using standard cutting technique.

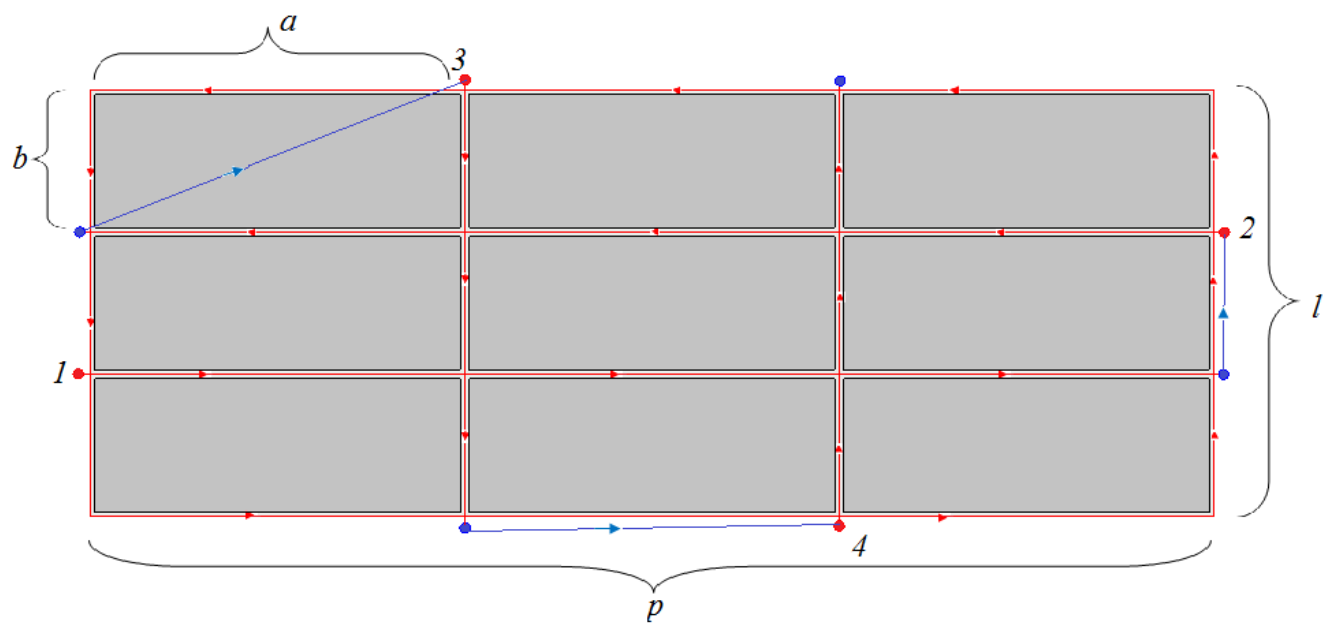

Fig. 2. The example of multi-contour cutting technique application with common cut for rectangle parts.

The proposed method of common cut application and forming groups of the same type parts at the stage of nesting can be interpreted as a method of basic segments generation for following solving of Generalized Segment Continuous Cutting Problem (GSCCP). The use 
of proposed method at the solving of cutting optimization problem in turn leads to reducing of cutting parameters values $-L_{o n}$ and $N_{p t}$, that leads to reducing of cost cutting objective function $-F_{\cos t}$. The proposed method is also effective for objective function of cutting time $T_{\mathrm{c} u t}$.

Below the computing experiments of proposed method application for cutting tool path generation compared with standard cutting technique.

\section{Computing experiments}

The performance evaluation of multi-contour technique application for selected group of parts compared with standard cutting technique application was completed at the example of two nesting maps contained real parts. The examples of nesting maps were taken from open library CPPLib [18]. As a base of compared the algorithm CCP-Relax is used for cutting path generation using standard cutting technique [19].

Fig. 3, 4 presents nesting maps for which the cutting tool path is generated by using standard cutting technique (fig.3a, 4a) and multi-contour cutting technique with common cut (fig. 3b, 4b).

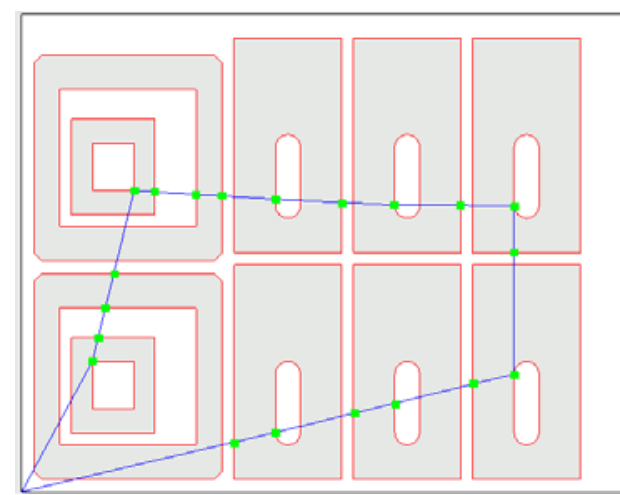

a)

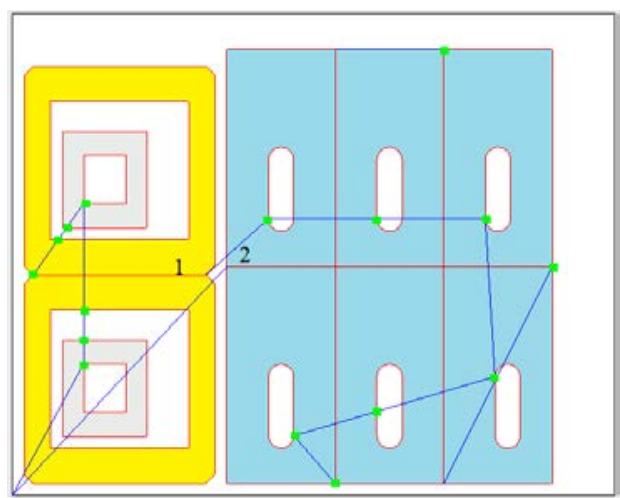

b)

Fig. 3. The cutting path problem solving for nesting map №1 using algorithm CCP-Relax (a) and in the case of multi-contour cutting with using of common cut (b)

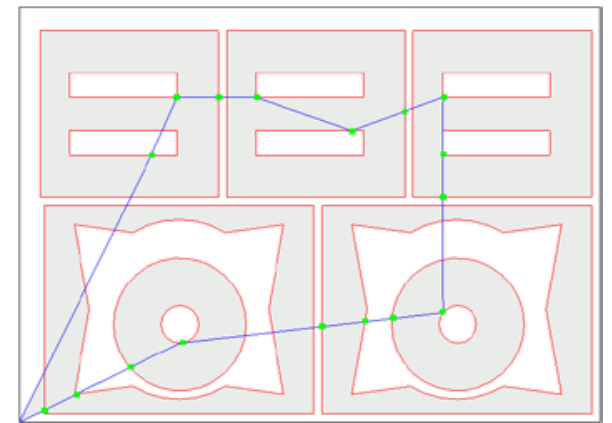

a)

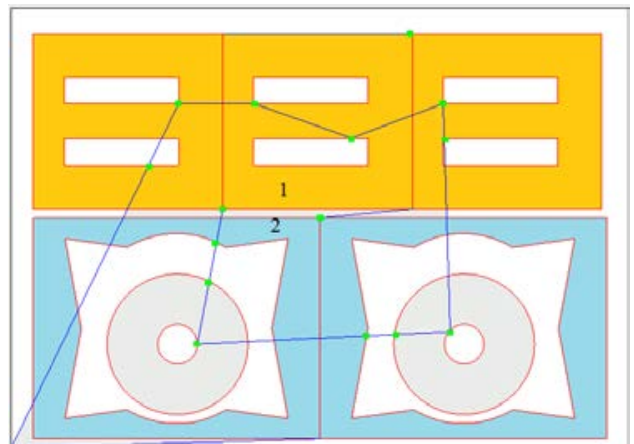

b)

Fig. 4. The cutting path problem solving for nesting map №2 using algorithm CCP-Relax (a) and in the case of multi-contour cutting technique with using of common cut (b) 
Fig.3b,4b present parts combined in «blocks» (two «blocks» are highlighted for each nesting map, each «block» is highlighted by yellow and blue), for each «block» the contour cutting is implemented using multi-contour cutting with common cut which is proposed in this article. Table 1 presents results of parameters values calculation $-L_{o n}$ and $N_{p t}$. The values of $L_{o n}$ and $N_{p t}$ parameters are reduced by application of proposed method, which finally leads to reduce the value of objective function $F_{\cos t}$. In spite of $L_{\text {off }}$ value is increased the value of $F_{\cos t}$ is less than $F_{\cos t}$ in the case of standard cutting technique use because values of $C_{o n}$ and $C_{p t}$ exceed significantly value of $C_{o f f}$. Fig 5 presents nesting maps with an increased number of rectangle contours in the case of multi-contour application. For the example the nesting map №1 was taken. The values of cutting parameters for the cases presented in fig. 5 are presented in table 1.

Table 1. The compare of cutting parameters values in terms of standard cutting and multi-contour cutting application.

\begin{tabular}{|l|c|c|c|c|c|c|c|c|}
\hline & $\begin{array}{c}\text { CCP- } \\
\text { Relax }\end{array}$ & $\begin{array}{l}\text { Multi- } \\
\text { contour }\end{array}$ & $\begin{array}{c}\text { CCP- } \\
\text { Relax }\end{array}$ & $\begin{array}{c}\text { Multi- } \\
\text { contour }\end{array}$ & $\begin{array}{c}\text { CCP- } \\
\text { Relax }\end{array}$ & $\begin{array}{c}\text { Multi- } \\
\text { contour }\end{array}$ & $\begin{array}{c}\text { CCP- } \\
\text { Relax }\end{array}$ & $\begin{array}{c}\text { Multi- } \\
\text { contour }\end{array}$ \\
\hline $\begin{array}{l}\text { Nesting } \\
\text { map }\end{array}$ & $\begin{array}{c}\text { №1 } \\
\text { fig.3a }\end{array}$ & $\begin{array}{c}\text { №1 } \\
\text { fig.3b }\end{array}$ & \multicolumn{2}{|c|}{$\begin{array}{c}\text { №1 } \\
\text { fig.5a }\end{array}$} & & $\begin{array}{c}\text { №1 } \\
\text { fig.5b }\end{array}$ & $\begin{array}{c}\text { №2 } \\
\text { fig.4a }\end{array}$ & $\begin{array}{c}\text { №2 } \\
\text { fig.4b }\end{array}$ \\
\hline $\begin{array}{l}\text { Number of } \\
\text { parts }\end{array}$ & \multicolumn{2}{|c|}{10} & \multicolumn{2}{|c|}{16} & \multicolumn{2}{|c|}{22} & \multicolumn{2}{|c|}{7} \\
\hline $\begin{array}{l}\text { Number of } \\
\text { contours }\end{array}$ & \multicolumn{2}{|c|}{20} & \multicolumn{2}{|c|}{32} & \multicolumn{2}{|c|}{44} & \multicolumn{2}{|c|}{17} \\
\hline$L_{\text {off }}, \mathrm{m}$ & 1,15 & 1,49 & 1,7 & 2,3 & 2,4 & 3,1 & 1,56 & 2,12 \\
\hline$L_{\text {on }}, \mathrm{m}$ & 7,33 & 6,2 & 11,6 & 9,2 & 15,8 & 12,11 & 9,87 & 9,22 \\
\hline$N_{p t}$ & 20 & 16 & 32 & 25 & 44 & 34 & 17 & 15 \\
\hline
\end{tabular}

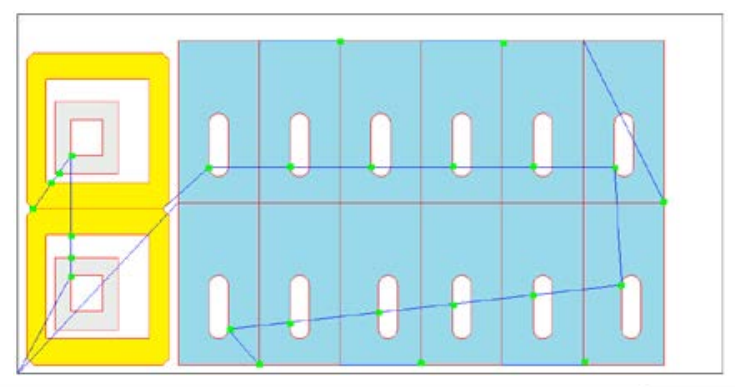

a)

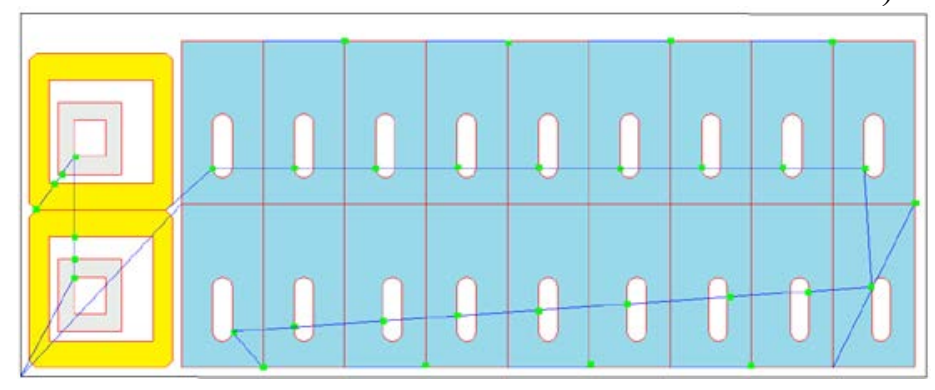

b)

Fig. 5. The example of nesting map №1 with increased number of rectangle contours in «block» 
The cutting cost is calculated by method presented in [20]. The calculation of cutting cost is performed using the example of contours cutting from titanium (3.7034) sheet with various thicknesses on the $\mathrm{CNC}$ laser equipment $\left(\mathrm{CO}_{2}\right)$. The results of $F_{\cos t}$ calculation presented in table 2 .

Table 2. The compare of cutting cost values in terms of standard cutting and multi-contour cutting application.

\begin{tabular}{|c|c|c|c|c|c|c|c|c|}
\hline & $\begin{array}{l}\text { CCP- } \\
\text { Relax }\end{array}$ & $\begin{array}{l}\text { Multi- } \\
\text { contour }\end{array}$ & $\begin{array}{l}\text { CCP- } \\
\text { Relax }\end{array}$ & $\begin{array}{l}\text { Multi- } \\
\text { contour }\end{array}$ & $\begin{array}{l}\text { CCP- } \\
\text { Relax }\end{array}$ & $\begin{array}{l}\text { Multi- } \\
\text { contour }\end{array}$ & $\begin{array}{l}\text { CCP- } \\
\text { Relax }\end{array}$ & $\begin{array}{l}\text { Multi- } \\
\text { contour }\end{array}$ \\
\hline Nesting map & $\begin{array}{c}\text { №1 } \\
\text { fig.3a }\end{array}$ & $\begin{array}{c}\text { №1 } \\
\text { fig. } 3 b\end{array}$ & & $\begin{array}{c}\text { №1 } \\
\text { fig. } 5 \mathrm{a}\end{array}$ & & $\begin{array}{c}\text { №1 } \\
\text { fig.5b }\end{array}$ & $\begin{array}{c}№ 2 \\
\text { fig.4a }\end{array}$ & $\begin{array}{c}\text { №2 } \\
\text { fig.4b }\end{array}$ \\
\hline \multicolumn{9}{|c|}{$3.7034, \Delta=1 \mathrm{~mm}$} \\
\hline$F_{\cos t}$, rub & 210,8 & 175,3 & 334,8 & 264,6 & 457,6 & 352,3 & 247 & 227,9 \\
\hline \multicolumn{9}{|c|}{$3.7034, \Delta=2 \mathrm{~mm}$} \\
\hline$F_{\cos t}$, rub & 331,7 & 275,8 & 526,8 & 416,1 & 719,8 & 553,8 & 391,4 & 361,1 \\
\hline \multicolumn{9}{|c|}{$3.7034, \Delta=3 \mathrm{~mm}$} \\
\hline$F_{\cos t}$, rub & 964,3 & 803 & 1531,0 & 1209,2 & 2091,2 & 1607,3 & 1157,5 & 1069,1 \\
\hline \multicolumn{9}{|c|}{$3.7034, \Delta=5 \mathrm{~mm}$} \\
\hline$F_{\cos t}$, rub & 3050,5 & 2551 & 4838,8 & 3825,8 & 6604,2 & 5071,2 & 3787,5 & 3510,1 \\
\hline
\end{tabular}

Tables 1 and 2 show that increasing of parts number in «block» (fig.5) the values of $L_{o n}$ and $N_{p t}$ are reduced compared with parameters values in the case of fewer common cuts (fig.3b). The value of cutting cost $F_{\cos t}$ is also reduced to $23 \%$ compared with standard cutting (CPP-Relax algorithm) in the case of increasing the parts in «block».

\section{Conclusions}

1. The method for generating groups of the same parts and multi-contour cutting use based on common cut application for rectangle parts with various dimensions at the stage of nesting for $\mathrm{CNC}$ sheet equipment is proposed;

2. Proposed method allows to reduce values of cutting parameters $-L_{o n}$ and $N_{p t}$ compared with standard cutting technique application what is confirmed by computing experiments (table 1). With the result that the value of objective function $F_{\cos t}$ is reduced too (table 2). This method is also effective for calculation of objective function $T_{\mathrm{c} u t}$. Proposed method is useful and effective at solving of optimization cutting problem for high-cost sheet metal (for example, titanium) at CNC technological equipment because a reusable scrap is generated. Fig. 3, 5 show that material saving is up to $9 \%$ compared with use of standard cutting technique during design of NC program;

3. The results of cutting cost calculation are given by the example of two nesting maps including real parts. The examples of nesting maps were taken from open library of test examples - CPPLib. It is shown that with increasing of common cuts numbers on the nesting map the values of cutting cost are reduced.

4. Thus the application of multi-contour cutting with common cut is useful in solving of cutting path problem during generation of $\mathrm{NC}$ program for $\mathrm{CNC}$ equipment. 
This research was supported by Ministry of Science and Higher Education of the Russian Federation, state contract № 075-03-2020-582/4.

\section{References}

1. A. Petunin, AIP: proceedings inter. conf. 1690, 060002 (2015)

2. M.A. Verhoturov, P.Yu. Tarasenko, Vestnik UGATU, 10, №2 (27), pp.123-130 (2008)

3. J. Hoeft, U.S. Palekar, IIE Transactions, 29 (9), pp.719 - 731 (1997).

4. R. Dewil, P. Vansteenwegen, D. Cattrysse, International Journal of Advanced Manufacturing Technology, 87(5), pp. 1865 - 1884 (2016).

5. A.A. Petunin, C. Stylios, IFAC-PapersOnLine, 49(12), pp. 23 - 28(2016)

6. A.G. Chentsov,. M.Yu. Khachai, D.M. Khachai, Proceedings of the Steklov Institute of Mathematics. pp. 38-46 (2016)

7. A.G. Chentsov,. M.Yu. Khachai, D.M. Khachai. IFAC-PapersOnLine. 49(12), pp.651655 (2016)

8. W. Yang, Y. Zhao, J. Jie, W. Wang. Advanced Material Reaesrch. pp. 373-377 (2010)

9. N. Ascheuer, M. Jiinger, G. Reinelt. Computational Optimization and Applications.17(1), pp. 61-84 (2000)

10. R. Dewil, P. Vansteenwegen, D. Cattrysse, T. Vossen, M. Laguna. International Journal of Production Research, 53(6), pp. 1761-1776 (2015)

11. Y. Jing, C. Zhige, Advanced Materials Research, 796, pp. 454-457 (2013)

12. S. Lin, B. Kernighan, Operations Research, 21(2), pp. 498-516 (1973)

13. K. Castelino, R. D’Souza, P.K. Wright, Journal of Manufacturing Systems, 22(3), pp. 173-180 (2003)

14. K. Vicencio, B. Davis. I. Gentilini, IEEE/RSJ International Conference on Intelligent Robots and Systems, pp. 2985 - 2990 (2014)

15. S. Veeramani, D. Kumar, International Journal of Production Research, 36(7), pp. 1901-1916 (1998)

16. M. Lee, K. Kwon, International Journal of Production Research, 44(24), pp.5307-5326 (2006)

17. A.A. Petunin, E.G. Polishchuk, S.S. Ukolov, IFAC-PapersOnLine, 53(13), pp. 23202325 (2019)

18. A. Petunin, A. Khalyavka, M. Khachay, A. Kudriavtsev, P. Chentsov, E. Polishchuk, S. Ukolov, Lecture Notes in Computer Science, 12665 (2021)

19. A.A. Petunin, E.G. Polishchuk, S.S. Ukolov, IFAC-PapersOnLine, 52(13), pp. 23202325 (2019)

20. A.F. Tavaeva, A.A. Petunin, J. modeling, optimization and information technology, $\mathbf{4}(23)(2018)$ 